\title{
Pseudo-nondiffracting beams generated by radial harmonic functions
}

\author{
Joseph Rosen, Boaz Salik, and Amnon Yariv \\ California Institute of Technology, m/s 128-95 Pasadena, California 91125
}

Received April 20, 1995; revised manuscript received June 20, 1995; accepted June 22, 1995

\begin{abstract}
A Fourier hologram with the distribution of a radial harmonic function creates a nondiverging beam in the far field. The properties of this beam are analyzed and the beam is demonstrated. We also give a set of theorems that describe the relations between the hologram and the longitudinal distribution of the beam. (c) 1995 Optical Society of America
\end{abstract}

\section{INTRODUCTION}

A pseudo-nondiffracting beam (PNDB) is characterized by an almost constant intensity along a finite propagation distance and a beamlike shape in its transverse dimensions. In other words, the intensity distribution is distributed as a sword of light. There are several methods for achieving a PNDB. From the viewpoint of geometrical optics, a sword beam has been demonstrated by axicon. ${ }^{1}$ With diffraction theory, it has been shown that the Bessel beam ${ }^{2}$ and a beam diffracted from the holographic axilens $^{3}$ behave as PNDB's. Other kinds of PNDB have been obtained by numerical iterative methods., ${ }^{4,5}$ Multiplexing a few PNDB's has been achieved also by numerical iterative methods, ${ }^{6}$ and one-dimensional PNDB's have been found analytically ${ }^{7}$ and numerically. ${ }^{8}$

In this paper we propose a new PNDB that is general in the sense that in the limit, when its parameters go to infinity, it converges to the Bessel beam. It consists of four independent parameters, each affecting the beam's transverse width, sidelobe height, and longitudinal interval length. The proposed PNDB's are created by radial harmonic functions (RHF's) coded on Fourier holograms. The RHF can be used as a phase-only pupil filter for increasing the depth of focus of imaging systems, as demonstrated in Section 5. The relations between the hologram and the resulting beam are analyzed in Section 3. Knowing the mutual dependence between the holograms and the PNDB's enables us to shift and rotate the beams in space, as well as change their scale, by changing a few parameters of the holograms' distribution. These effects are also demonstrated in Section 5.

\section{BACKGROUND}

The original nondiffracting beam, also called the Bessel beam, ${ }^{2}$ is a solution, of the form $E(r, z)=$ $A \exp (j \beta z) J_{0}(\alpha r)$, to the free-space scalar-wave equation, in which $\alpha^{2}+\beta^{2}=k^{2}, k$ is the wave number, $J_{0}$ is the zero-order Bessel function, and $r, z$ are the cylindrical coordinates. This beam is interesting because its intensity distribution in the entire space is independent of the $z$ coordinate, and the transverse intensity profile has a beamlike shape.
It has been also shown ${ }^{9}$ that the Bessel beam can be obtained by illumination of an annular aperture, located at the rear focal plane (plane $P_{i}$ in Fig. 1) of a spherical lens. This phenomenon can be generalized if one assumes an arbitrary function $g\left(r_{i}, \theta_{i}\right)$, with circular symmetry [i.e., $\left.g\left(r_{i}, \theta_{i}\right)=g\left(r_{i}\right)\right]$, placed as a transparency distribution at plane $P_{i}$. Under the Fresnel approximation the complex amplitude distribution around the focal plane $P_{f}$ is ${ }^{5,10}$

$$
\begin{aligned}
u(r, z)= & \frac{\exp [j k(z+2 f)]}{j \lambda f} \int_{0}^{\infty} g\left(r_{i}\right) J_{0}\left(\frac{2 \pi r r_{i}}{\lambda f}\right) \\
& \times \exp \left(-\frac{j \pi z r_{i}^{2}}{\lambda f^{2}}\right) r_{i} \mathrm{~d} r_{i}
\end{aligned}
$$

where $k=2 \pi / \lambda, \lambda$ is the wave length, $f$ is the focal length of the lens, $r_{i}$ is the radial coordinate of plane $P_{i}$, and $(r, z)$ are the cylindrical coordinates beyond the lens, with the front focus as their origin. By substituting $r=0$ into Eq. (1) we see that there is a Fourier-transform relation between the longitudinal profile $u(r=0, z)$ around the front focal plane of the lens (plane $P_{f}$ ) and the square radial distribution of the field in the rear focal plane (plane $P_{i}$ ); i.e.,

$$
u(0, z)=\frac{\exp [j k(z+2 f)]}{j 2 \lambda f} \int_{0}^{\infty} g\left(\sqrt{\rho_{i}}\right) \exp \left(-\frac{j k z \rho_{i}}{2 f^{2}}\right) \mathrm{d} \rho_{i},
$$

where $\rho_{i}=r_{i}{ }^{2}$. The annular aperture, for instance, is represented by $g\left(r_{i}\right)=\delta\left(r_{i}-\alpha\right)$, and substituting it into Eq. (1) yields $u(r, z)=a J_{0}(k a r / f) \exp \left[j k z\left(1-a^{2} / 2 f^{2}\right)\right]$, i.e., the Bessel beam. The three-dimensional intensity distribution of the Bessel beam is independent of $z$ in the entire space $z>0$. However, since we need an infinite energy to implement the Bessel beam, in practice any truncation yields a PNDB whose intensity changes along its propagation. A real annular mask can yield only an approximation to the ideal Bessel beam in the sense that the peak intensity of this beam oscillates slightly around some constant value up to a point where the intensity drops significantly. ${ }^{2,9}$ Since we deal only with an approximation to the ideal nondiffracting beam, the Bessel beam is no longer the only option for obtaining a PNDB. We 


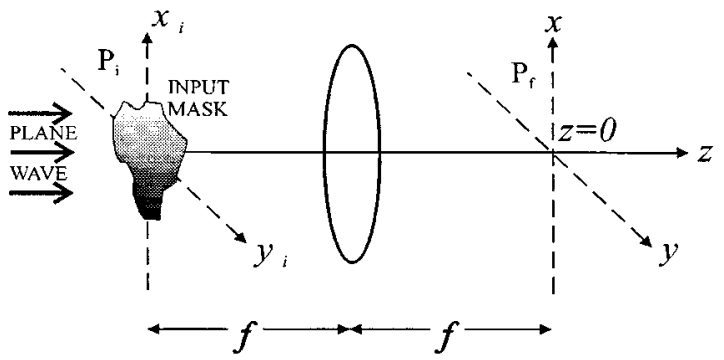

Fig. 1. Schematic system used to obtain the PNDB.

shall show that there are other functions that can be used as PNDB's with features sometimes superior to those of the Bessel beam. But before that, we list several general theorems that determine the relations between the input function and the longitudinal distribution of the beam.

\section{THEOREMS OF THE LONGITUDINAL FOURIER HOLOGRAM}

Before we introduce the theorems, let us define a transform, called the focal-space transform (FST), as

$$
\begin{aligned}
u(x, y, z)= & \operatorname{FST}\left\{g\left(x_{i}, y_{i}\right)\right\} \\
= & \frac{\exp [j k(z+2 f)]}{j \lambda f} \int_{-\infty}^{\infty} \int_{-\infty}^{\infty} g\left(x_{i}, y_{i}\right) \\
& \times \exp \left(\frac{j 2 \pi\left(x x_{i}+y y_{i}\right)}{\lambda f}-\frac{j \pi z\left(x_{i}^{2}+y_{i}^{2}\right)}{\lambda f^{2}}\right) \\
& \times \mathrm{d} x_{i} \mathrm{~d} y_{i} .
\end{aligned}
$$

$u(x, y, z)$ is the complex amplitude in the entire space around the focal plane $P_{f}$ resulting from the input distribution $g\left(x_{i}, y_{i}\right)$, and in fact, it is the Cartesian version of Eq. (1). The following theorems quantify the changes in the distribution behind the lens resulting from modification of the input function $g\left(x_{i}, y_{i}\right)$. The proofs of these theorems are given in Appendix A.

1. Complex-conjugate theorem: if $\operatorname{FST}\left\{g\left(x_{i}, y_{i}\right)\right\}=$ $u(x, y, z)$, then

$$
\operatorname{FST}\left\{g^{*}\left(x_{i}, y_{i}\right)\right\}=\exp (j 4 k f-j \pi) u^{*}(-x,-y,-z) .
$$

As a result of this theorem, one can see that replacing the input function by its complex conjugate flips the intensity distribution about its origin (the focal point).

2. Linear phase theorem: if $\operatorname{FST}\left\{g\left(x_{i}, y_{i}\right)\right\}=u(x, y$, $z)$, then

$$
\begin{aligned}
\operatorname{FST}\left\{g\left(x_{i}, y_{i}\right) \exp \left[j 2 \pi\left(d_{x} x_{i}+d_{y} y_{i}\right)\right]\right\} & \\
& =u\left(x-\lambda f d_{x}, y-\lambda f d_{y}, z\right) .
\end{aligned}
$$

Multiplying $g\left(x_{i}, y_{i}\right)$ by a linear phase function shifts the longitudinal beam laterally by distances $\left(\lambda f d_{x}, \lambda f d_{y}\right)$ that are directly related to the phase constants.

3. Quadratic phase theorem: if $\operatorname{FST}\left\{g\left(x_{i}, y_{i}\right)\right\}=$ $u(x, y, z)$, then

$$
\begin{aligned}
\operatorname{FST}\left\{g\left(x_{i}, y_{i}\right) \exp \left(j 2 \pi \alpha r_{i}{ }^{2}\right)\right\}= & \exp \left(j 4 \pi f^{2} \alpha\right) \\
& \times u\left(x, y, z-2 \lambda f^{2} \alpha\right) .
\end{aligned}
$$

Multiplying $g\left(x_{i}, y_{i}\right)$ by a quadratic phase function shifts the longitudinal beam along the $z$ axis by a distance $2 \lambda f^{2} \alpha$, which is directly related to the phase constant.

4. Lateral-shift theorem: if $\operatorname{FST}\left\{g\left(x_{i}, y_{i}\right)\right\}=u(x$, $y, z)$, then

$$
\begin{aligned}
& \operatorname{FST}\left\{g\left(x_{i}-d_{a}, y_{i}-d_{b}\right)\right\}=\exp (j k \chi) \\
& \times u(\bar{x} \sec \theta, \bar{y} \sec \varphi, \bar{z} \cos \theta \cos \varphi),
\end{aligned}
$$

where

$$
\chi=x \tan \theta+y \tan \varphi-\frac{z}{2}\left(\tan ^{2} \theta+\tan ^{2} \varphi\right)
$$

$\tan \theta=d_{a} / f$, $\tan \varphi=d_{b} / f$, and $(\bar{x}, \bar{y}, \bar{z})$ is the tiltedcoordinates system at an angle $\theta$ to the $y-z$ plane and $\varphi$ to the $x-z$ plane. This theorem states that when the input function is shifted laterally, the output beam is rotated around the focal point by angles $(\theta, \varphi)$, whose tangents are directly related to the lateral shift $\left(d_{a}, d_{b}\right)$. The longitudinal dimension of the rotated beam is stretched by the factor $\sec \theta \sec \varphi$, and the lateral dimensions $(\bar{x}, \bar{y})$ are shrunk by the factors $\cos \theta$ and $\cos \varphi$, respectively.

$$
\begin{aligned}
& \text { 5. Similarity Theorem: if } \operatorname{FST}\left\{g\left(r_{i}\right)\right\}=u(x, y, z) \text {, } \\
& \text { then } \\
& \operatorname{FST}\left\{g\left(s r_{i}\right)\right\}=\frac{\exp \left[j k z\left(1-s^{-2}\right)\right]}{s^{2}}\left(\frac{r}{s}, \frac{z}{s^{2}}\right) .
\end{aligned}
$$

Shrinking (stretching) the size of the input function by $s$ times increases (decreases) the longitudinal dimension of the beam by $s^{2}$ times, whereas the lateral dimension increases (decreases) by only $s$ times.

The next theorem does not belong to the previous longitudinal Fourier hologram theorems, because in this theorem we consider the effect of shifting the hologram along the $z$ axis out of the rear focal plane. Therefore the hologram can no longer be considered a longitudinal Fourier hologram, and none of the previous theorems apply.

6. Longitudinal-shift theorem: if $\operatorname{FST}\left\{g\left(x_{i}, y_{i}\right)\right\}=$ $u(x, y, z)$, then displaying the input mask at an arbitrary distance $d$ from the lens yields a longitudinal distribution $\bar{u}(x, y, z)$, given by

$$
\bar{u}(x, y, z)=A(x, y, z) u(\tilde{x}, \tilde{y}, \tilde{z})
$$

where

$$
\begin{aligned}
(\tilde{x}, \tilde{y}, \tilde{z}) & =\frac{f^{2}}{z(f-d)+f^{2}}(x, y, z), \\
A(x, y, z) & =\frac{f^{2} \exp \left[j k\left(z+d-f+\frac{(f-d) r^{2}-2 f^{2} z}{2\left[z(f-d)+f^{2}\right]}\right)\right]}{z(f-d)+f^{2}} .
\end{aligned}
$$

When the distance between $g\left(x_{i}, y_{i}\right)$ and the lens is changed from $f$ to another distance $d$, the longitudinal field is transformed to the $(\tilde{x}, \tilde{y}, \tilde{z})$ coordinates, and the function $A(x, y, z)$ induces attenuation along the $z$ axis 
compared with the case of $d=f$. Of course, when $d=f$, then $\bar{u}(x, y, z)=u(x, y, z)$.

These theorems enable one to shift, scale, and tilt the PNDB in the entire space. Another application for them is the creation of arbitrary twisted focal lines, socalled snake beams, presented by us (Rosen and Yariv) in Ref. 11.

\section{PSEUDO-NONDIFFRACTING BEAMS OBTAINED BY RADIAL HARMONIC FUNCTIONS}

In this section we introduce the new PNDB and study its characteristics. After presenting the list of general requirements for any $\mathrm{PNDB}$ and the RHF that realizes it, we analyze the axial and lateral features of the resulting beam.

\section{A. Radial Harmonic Functions}

A beam $u(r, z)$ may be considered a PNDB if the following set of three equations is satisfied:

$$
\begin{array}{clrl}
|u(0, z)|^{2} & =\text { const., } & & \forall z \in \Delta z, \\
\max _{r}\{|u(r, z)|\} & =|u(0, z)|, & \forall z \in \Delta z, \\
\lim _{r \rightarrow \infty}|u(r, z)|=0, & \forall z \in \Delta z, \\
\text { even if } \int_{0}^{R}|u(r, z)|^{2} r \mathrm{~d} r \underset{R \rightarrow \infty}{\longrightarrow} \infty .
\end{array}
$$

Equation (4a) represents the requirement that the peak intensity of the beam stay constant along some interval $\Delta z$. Equations (4b) and (4c) provide the beamlike shape to the transverse intensity distribution of the beam at any point $z$ inside the interval $\Delta z$. By Eq. (4b) we guarantee that the maximum of the lateral distribution is obtained on the $z$ axis. Attenuation of the beam's tails to zero far away from the $z$ axis, even for an infinite energy beam, is achieved by Eq. (4c).

Using the theory of stationary phase approximation, ${ }^{12}$ we obtain the following RHF, which approximately satisfies Eqs. (4):

$$
g_{p}\left(r_{i}\right)=\left(r_{i} / a\right) \exp \left[j 2 \pi\left(r_{i} / b\right)^{p}\right], \quad p \geq 4,
$$

where $p$ is a real number greater than 4 , in order to avoid singularity of $g_{p}\left(r_{i}\right)$ at the origin, and $a$ and $b$ are real numbers. The special case of $p=4$ is especially interesting, because it is the only case of a pure (fourth-order) phase function in this family of functions. Consequently, the light is not absorbed when it passes through a mask with the transparency function $g_{p=4}\left(r_{i}\right)$. For this reason we also mention some of our results specifically for $p=4$ and demonstrate our results with $g_{p=4}\left(r_{i}\right)$. Note that Eq. (5) is a nonunique solution to Eqs. (4), since the solution $g\left(r_{i}\right)=\delta\left(r_{i}-\gamma\right)$, ( $\gamma$ is real) also satisfies Eqs. (4). This solution leads to the well-known Bessel beam.

\section{B. Axial Distribution}

In order to verify that Eqs. (4) are satisfied, we first check Eq. (4a). Substituting the function $g_{p}\left(r_{i}\right)$ [Eq. (5)] into Eq. (2) yields

$$
\begin{aligned}
u(0, z)= & \frac{\exp [j k(z+2 f)]}{j \lambda f a^{(4-p) / 2}} \int_{0}^{\infty} r_{i}^{(p-2) / 2} \exp \left(\frac{j 2 \pi r_{i}^{p}}{b^{p}}\right. \\
& \left.-\frac{j \pi z r_{i}^{2}}{\lambda f^{2}}\right) \mathrm{d} r_{i}
\end{aligned}
$$

The stationary-phase approximation yields the following solution for Eq. (6):

$$
\begin{aligned}
u(0, z) \cong & \frac{\exp [j k(z+2 f)+j \pi / 4]}{j \lambda f} \sqrt{\frac{b^{p}}{p(p-2) a^{p-4}}} \\
& \times \exp \left(-j \pi \gamma z^{p /(p-2)}\right), \quad z>z_{1},
\end{aligned}
$$

where

$$
\begin{aligned}
\gamma & =(p-2)\left(\frac{b^{2}}{p \lambda f^{2}}\right)^{p /(p-2)} \underset{p=4}{=} \frac{b^{4}}{8 \lambda^{2} f^{4}}, \\
z_{1} & =\frac{p \lambda f^{2}}{b^{2}(2 p-4)^{(p-2) / p}} \underset{p=4}{=} \frac{2 \lambda f^{2}}{b^{2}} .
\end{aligned}
$$

Indeed, from a certain point the intensity along the $z$ axis is approximately constant, which means that Eq. (4a) is satisfied in the interval $\Delta z=\left[z_{1}, \infty\right]$. The phase distribution of $u(0, z)$ varies from a quadratic phase for $p=4$ down to a linear phase in the limit of $p \rightarrow \infty$. The starting point $z_{1}$ is calculated on the basis of the condition that the phase value $\mu(r)$ of the integrand of Eq. (6) at the significant stationary point $r_{s}$ be $\pi / 2$. This point is far enough ( $z$ is large enough) from the zero point $\left(r_{i}=0\right.$, another stationary point but not a contributing one), that it contributes fully to the integral value. Formally, the condition that should be satisfied is

$$
k \mu\left(r_{s}\right)=\frac{2 \pi r_{s}{ }^{p}}{b^{p}}-\frac{\pi z_{1} r_{s}^{2}}{\lambda f^{2}}=\frac{\pi}{2},
$$

where

$$
r_{s}=\left(\frac{b^{p} z}{p \lambda f^{2}}\right)^{1 /(p-2)}
$$

is the stationary point obtained as the solution of the equation $d \mu / d r_{i}=0$.

Practically, an infinite function $g\left(r_{i}\right)$ cannot be implemented, and when the finite radius $R_{0}$ of the input mask (the fourth parameter of the RHF's, in addition to $a, b$, and $p$, assuming from now on that $R_{0}>b$ ) is taken into account, the absolute value of $u(0, z)$ oscillates around a constant level along a finite interval $\Delta z=z_{2}-z_{1} \approx z_{2}$. This response is shown by the solid curve in Fig. 2 for the parameters $p=4, b=256$ pixels, and $R_{0}=512$ pixels. For comparison, the axial response of a clear-aperture lens of diameter $2 b$ is shown by the dashed curve. The value of the bend point $z_{2}$ is calculated on the basis of the condition that the stationary point is located close to the edge of the aperture at the last point at which it contributes fully to the integral result [Eq. (6)] (i.e., $r_{s \mid z=z_{2}}=R_{0}-\Delta$, where $\Delta$ is the width in which the phase changes by $\pi / 2$ from the phase at $r_{s \mid z=z_{2}}=R_{0}$ ). If we use Eq. (8a), $z_{2}$ is given by

$$
\begin{aligned}
z_{2} & \cong \frac{p \lambda f^{2} R_{0}^{p-3}}{b^{p}}\left[R_{0}-\sqrt{\frac{(p-2) b^{p}}{2 p R_{0}{ }^{p-2}}}\right] \\
& =\frac{2 \lambda f^{2}\left(2 R_{0}^{2}-b^{2}\right)}{b^{4}} .
\end{aligned}
$$




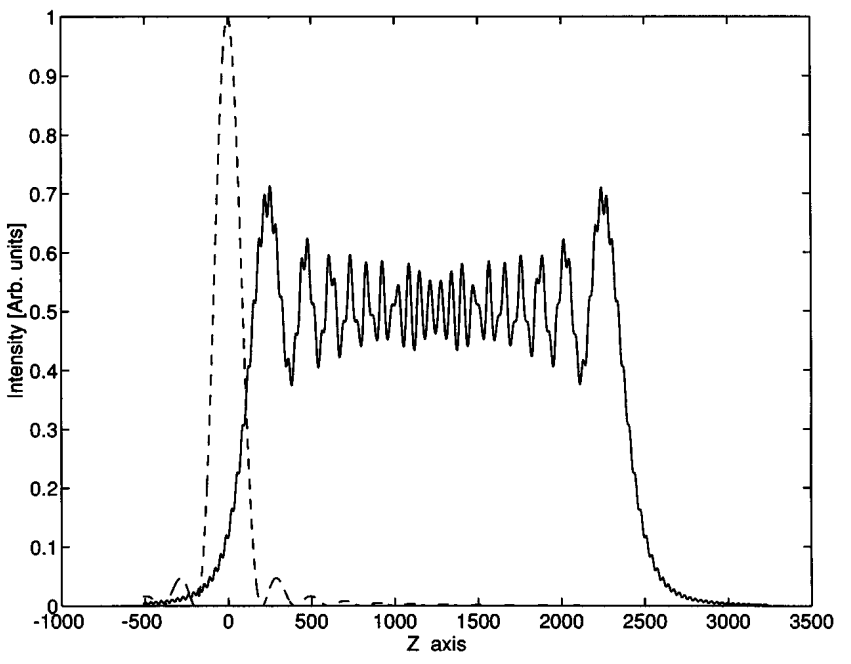

Fig. 2. Computer-simulated axial intensity distribution of the PNDB (solid curve) and of the ordinary focused beam (dashed curve).

From Eq. (9) it is seen that one can increase the longitudinal interval $\Delta z$ by increasing the parameters $R_{0}$, or $p$ or by decreasing $b$.

At this point, when the upper limit of the integral in Eq. (6) is $R_{0}$, we can give a better approximation for $u(0, z)$ than that of Eq. (7) (see Ref. 12):

$$
\begin{aligned}
u(0, z) \cong & \frac{\exp [j k(z+2 f)]}{j \lambda f \sqrt{a^{p-4}}}\left\{\sqrt { \frac { b ^ { p } } { p ( p - 2 ) } } \operatorname { e x p } \left[-j \gamma z^{p /(p-2)}\right.\right. \\
& +j \pi / 4]-\frac{\lambda f^{2}\left(2 R_{0}\right)^{(p-4) / 2}}{2 \pi\left(\lambda f^{2} p R_{0}^{p-2} b^{-p}-z\right)} \\
& \left.\times \exp \left(\frac{-j k R_{0}^{2} z}{2 f^{2}}+\frac{j 2 \pi R_{0} p}{b^{p}}\right)\right\}, \quad z_{1}<z<z_{2} .
\end{aligned}
$$

The second term of Eq. (1) is dependent on $z$ through the amplitude and the linear phase. The case of $p=4$ is slightly different from the others since the term obtained as a result of the lower limit $\left(r_{i}=0\right)$ is not negligible. Therefore the amplitude along the $z$ axis for $p=4$ is

$$
\begin{aligned}
u(0, z)_{\left.\right|_{p=4}} \cong & \frac{\exp [j k(z+2 f)]}{j \lambda f}\left[\frac{b^{2}}{2 \sqrt{2}} \exp \left(-j \gamma z^{2}+j \pi / 4\right)\right. \\
& -\frac{\lambda f^{2} / 2 \pi}{\left(4 \lambda f^{2} R_{0}^{2} / b^{4}\right)-z} \exp \left(\frac{-j k R_{0}^{2} z}{2 f^{2}}\right. \\
& \left.\left.+\frac{j 2 \pi R_{0}^{4}}{b^{4}}\right)-\frac{\lambda f^{2}}{2 \pi z}\right], \quad z_{1}<z<z_{2}
\end{aligned}
$$

The second and third terms of Eq. (11) are the main source for the oscillations in the solid curve of Fig. 2. These oscillations increase near $z_{1}$ as a result of the third term and near $z_{2}$ as a result of the second term. The two terms imply a way for one to perform apodization on the original input mask in order to eliminate these oscillations ${ }^{13}$; however, that subject is beyond the scope of this paper.

\section{Lateral Distribution}

Next we consider the lateral features of the PNDB obtained from the RHF. We obtain the lateral distribu- tion of the beam near the axis by substituting Eq. (5) into Eq. (1) and calculating Eq. (1) for small $r(r \ll$ $\left.\lambda f R_{0}{ }^{p-1} / b^{p}\right)$, in the same manner as Eq. (6) is calculated (assuming infinite aperture width):

$$
\begin{aligned}
u(r, z) \cong & \frac{\exp [j k(z+2 f)+j \pi / 4]}{j \lambda f} J_{0}\left[\frac{2 \pi r}{\lambda f}\left(\frac{b^{p} z}{p \lambda f^{2}}\right)^{1 /(p-2)}\right] \\
& \times \sqrt{\frac{b^{p}}{p(p-2) a^{p-4}}} \exp \left[-j \gamma z^{p /(p-2)}\right], \quad z \in \Delta z .
\end{aligned}
$$

Observing Eq. (12), we conclude that when $p \rightarrow \infty$ the beam becomes the Bessel beam. This beam is equivalent to a Bessel beam obtained by illumination of an annular function $g\left(r_{i}\right)=\delta\left(r_{i}-b\right)$. The ratio $b / a$ determines whether the energy of the beam increases $(b / a>1)$ or decreases $(b / a<1)$ with increasing $p$. In that sense this PNDB is general and contains the solution of the Bessel beam.

From Eq. (12) we can also learn that the full width at half-maximum (FWHM) of the lateral pulse intensity is

$$
W_{P}(z) \cong \frac{2.26}{2 \pi}\left(\frac{p \lambda^{p-1} f^{p}}{b^{p} z}\right)^{1 /(p-2)} \underset{p=4}{=} 0.72 \frac{f^{2}}{b^{2}} \sqrt{\frac{\lambda^{3}}{z}} .
$$

The FWHM decreases along the optical axis as $1 / z^{1 /(p-2)}$, or, in other words, the FWHM falls off as $1 / \sqrt{z}$ for $p=4$ and becomes independent of $z$ for $p \rightarrow \infty$. Substituting Eq. (7b) into Eq. (13), we see that at $z_{1}$ the FWHM is independent of the radius $R_{0}$ and is given by

$$
W_{P}\left(z_{1}\right) \cong \frac{2.26 \lambda f}{2 \pi b}(2 p-4)^{1 / p} \underset{p=4}{=} 0.51 \frac{\lambda f}{b} .
$$

This FWHM is approximately equal to the FWHM of a focal spot obtained by illumination of a lens of focal length $f$ and a clear aperture with diameter $2 b$. Substituting Eq. (9) into Eq. (13) and assuming that $R_{0} \gg b$, we see that at $z_{2}$ the FWHM is independent of $p$ or $b$ and is given by

$$
W_{P}\left(z_{2}\right) \cong \frac{0.36 \lambda f}{R_{0}},
$$

which is approximately equal to the FWHM of a focal spot obtained by illumination of a lens of focal length $f$ and a clear aperture with diameter $2.8 R_{0}$.

We conclude that the RHF optical system has the minimum resolution (at $z_{1}$ ) of a clear-aperture system with pupil diameter $2 b$. Moreover, increasing the diameter of the RHF does not change this resolution but increases the system's depth of focus and decreases the focal spot's width along the extended depth of focus. Furthermore, the maximum resolution (at $z_{2}$ ) is $40 \%$ better than that of a clear-aperture system with pupil diameter $2 R_{0}$.

At this point we are able to compare the PNDB with the Gaussian beam. The Rayleigh depth of focus is $\Delta z_{G}=$ $4 W^{2} / \lambda$, where $W$ is the FWHM of the beam. The ratio between the longitudinal interval of the PNDB, given in Eq. (9), and the depth of focus for the Gaussian beam is

$$
\frac{\Delta z_{p}}{\Delta z_{G}}=\frac{p \lambda^{2} f^{2} R_{0}^{p-2}}{4 W^{2} b^{p}} .
$$

When the PNDB is compared with a Gaussian beam with 
$W=W_{P}\left(z_{1}\right)$, the ratio becomes

$$
\frac{\Delta z_{P}}{\left.\Delta z_{G}\right|_{W=W_{P}\left(z_{1}\right)}}=\frac{1.93 p}{(2 p-4)^{2 / p}}\left(\frac{R_{0}}{b}\right)^{p-2} \underset{p=4}{\cong} 4\left(\frac{R_{0}}{b}\right)^{2} .
$$

When the PNDB is compared with a Gaussian beam with $W=W_{P}\left(z_{2}\right)$, the ratio becomes

$$
\frac{\Delta z_{P}}{\left.\Delta z_{G}\right|_{W=W_{P}\left(z_{2}\right)}}=1.93 p\left(\frac{R_{0}}{b}\right)^{p} \underset{p=4}{\cong} 8\left(\frac{R_{0}}{b}\right)^{4} .
$$

From these results we can conclude that the PNDB always has a longer focal depth than does the Gaussian beam if $R_{0}>b$, even with the wider waist of $W=W_{P}\left(z_{1}\right)$. This superiority increases with $p$ and with the ratio $R_{0} / b$. Note that in the case of the Gaussian beam, decreasing the system aperture (or the numerical aperture), say by $x$ times, causes an increase in the depth of focus by $x^{2}$ and a decrease in the lateral resolution by $x$ times. On the other hand, in our PNDB for $p=4$, decreasing the system aperture by the same amount does not change the resolution of the beam (as long as $R_{0}>b$ ), and it decreases the depth of focus by $x^{2}$. Moreover, decreasing the parameter $b$ by $x$ times decreases the resolution by the same amount but increases the depth of focus by $x^{4}$. The behavior of this PNDB is completely different from that of an ordinary beam, and trying to manifest a distance analogous to the Rayleigh distance yeilds [by substituting Eqs. (14) and (15) into Eq. (9) and choosing $p=4]$

$$
\Delta z_{P} \cong \frac{16\left[W_{P}\left(z_{1}\right)\right]^{4} \mathrm{NA}^{2}}{\lambda^{3}} \cong \frac{8.3\left[W_{P}\left(z_{1}\right)\right]^{4}}{\lambda\left[W_{P}\left(z_{2}\right)\right]^{2}},
$$

where $\mathrm{NA}=D / f$ is the numerical aperture.

Next we consider the asymptotic behavior of the lateral intensity distribution inside the interval $\Delta z$ and prove Eq. (4c). One obtains this lateral distribution by substituting $g_{p}\left(r_{i}\right)$ into Eq. (1) and approximating asymptotically the Bessel function: $J_{0}\left(2 \pi r r_{i} / \lambda f\right) \cong$ $\cos \left(2 \pi r r_{i} / \lambda f\right) / \sqrt{r r_{i}}$. Under this approximation the beam distribution is

$$
\begin{aligned}
u(r, z)_{\mid r \gg \lambda f R_{0}{ }^{p-1 / b^{p}}}= & \frac{\exp [j k(z+2 f)]}{j \lambda f \sqrt{r}} \int_{0}^{\infty} r_{i}^{(p-3) / 2} \\
& \times \exp \left(\frac{j 2 \pi r^{p}}{b^{p}}-\frac{j \pi z r_{i}^{2}}{\lambda f^{2}}\right) \\
& \times \cos \left(\frac{2 \pi r r_{i}}{\lambda f}\right) \mathrm{d} r_{i}
\end{aligned}
$$

On the basis of the stationary-phase approximation, ${ }^{12}$ the lateral intensity, for $r \gg \lambda f R_{0}{ }^{p-1} / b^{p}$, at $z=0$, is related as $1 / r \bar{r}_{s}$, where $\bar{r}_{s}$ is the stationary point of the phase distribution of the integrand in Eq. (20), related to $r$ as $r^{1 /(p-1)}$. Therefore we conclude that

$$
I(r, z) \underset{r \rightarrow \infty}{\propto} r^{-p /(p-1)} \quad \text { for } z=0 .
$$

The lateral intensity is attenuated maximally as $r^{-4 / 3}$ for $p=4$ and as $r^{-1}$ for $p \rightarrow \infty$, which is exactly the same attenuation as for the Bessel beam. The plane $z=0$, however, is not contained in the interval of interest, $\Delta z$.
The lateral intensity, for $r \gg \lambda f R_{0}{ }^{p-1} / b^{p}$ and $z>0$, is related as

$$
\begin{aligned}
& I(r, z) \underset{r \rightarrow \infty}{\propto} r^{-1} \bar{r}_{s}{ }^{p-3}\left[\frac{(p-1) p \lambda}{b^{p}} \bar{r}_{s}{ }^{p-2}-\frac{2 z}{f^{2}}\right]^{-1}, \\
& \forall z \in \Delta z,
\end{aligned}
$$

where

$$
\bar{r}_{s}=r\left[\left(\frac{p \lambda f}{b^{p}} r^{p-2}\right)^{1 /(p-1)}-\frac{2 z}{f(p-1)}\right]^{-1} .
$$

From Eq. (22) we realize that along the beam propagation the energy and radius of the sidelobes increase. Equation 4(c), however, is still valid along the whole range $\Delta z$, since for $r \rightarrow \infty$ the terms with $z$ in Eqs. (22) and (22a) become negligible. Note that Eq. (4c) is satisfied even for infinite-aperture masks $\left(R_{0} \rightarrow \infty\right)$; i.e., the attenuation of the lateral amplitude toward zero exists even if the total energy at every plane is infinite.

The width of the beam's sidelobes along the interval $\Delta z$ can be also estimated by observation of Eq. (20). The stationary point is obtained as a solution of the following equation:

$$
\frac{\mathrm{d} \mu}{\mathrm{d} r_{i}}=\frac{p r_{i}^{p-1}}{b^{p}}-\frac{z r_{i}}{\lambda f^{2}}+\frac{r}{\lambda f}=0
$$

In order to estimate the width of the sidelobes, we calculate the maximum value of $r$ that still yields a positive solution for Eq. (23):

$$
r_{m}=\frac{p-2}{p-1}\left(\frac{z^{p-1} b^{p}}{\lambda f^{p} p(p-1)}\right)^{1 /(p-2)} \underset{p=4}{=} \frac{b^{2} z^{3 / 2}}{3 f^{2} \sqrt{3 \lambda}} .
$$

This is approximately the half-width of the beam's sidelobes as function of $z$. At the two edges of $\Delta z$ the full widths, for $p=4$, are

$$
2 r_{m}\left(z_{1}\right)=\frac{4 \sqrt{2} \lambda f}{3 \sqrt{3} b}, \quad 2 r_{m}\left(z_{2}\right)=\frac{16 \lambda f R_{0}^{3}}{3 \sqrt{3} b^{4}} .
$$

Finally, we verify that Eq. (4b) is satisfied. In order to do so we have to show that the intensity at any point $(r, z)$ is less than the intensity at the point $(0, z)$, for any $z \in \Delta z$; i.e.,

$$
|u(r, z)|<|u(0, z)|, \quad \forall z \in \Delta z .
$$

$u(0, z)$ is given in Eq. (7), and $u(r, z)$, for small $r$, is given in Eq. (12). Since $\left|J_{0}(r>0)\right|<1$, Eq. (26) is satisfied in the range of small $r$. When $r$ increases, the intensity at every plane $z \in \Delta z$ falls off asymptotically as given in Eq. (22), and it is clear that Eq. (26) is satisfied for any $r$.

Transverse intensity cross sections of the beam, at various distances from the focal point, are shown in Fig. 3 for $p=4, p=128$ pixels, and $R_{0}=256$ pixels. These curves validate and demonstrate the theory and the conclusions of the lateral behavior of the PNDB as described above. 


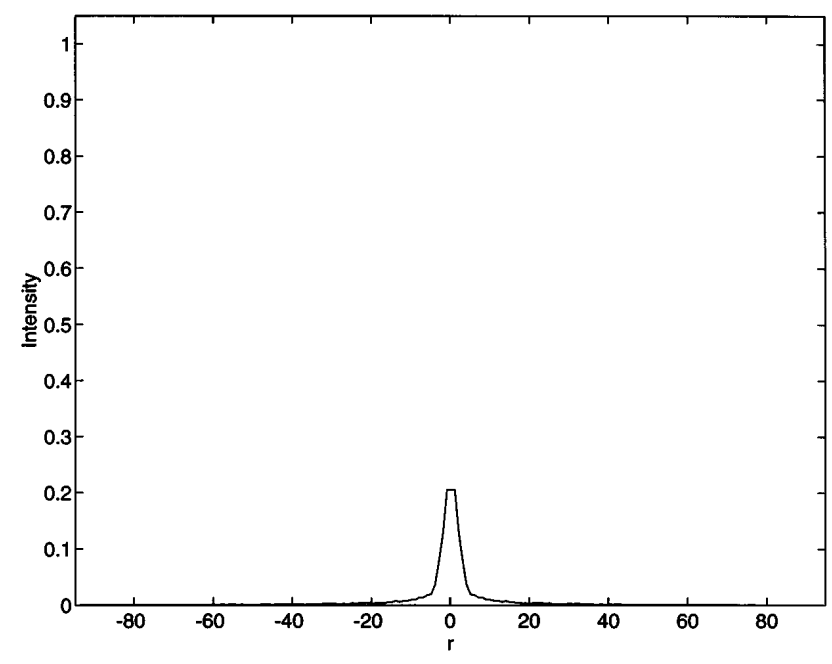

(a)

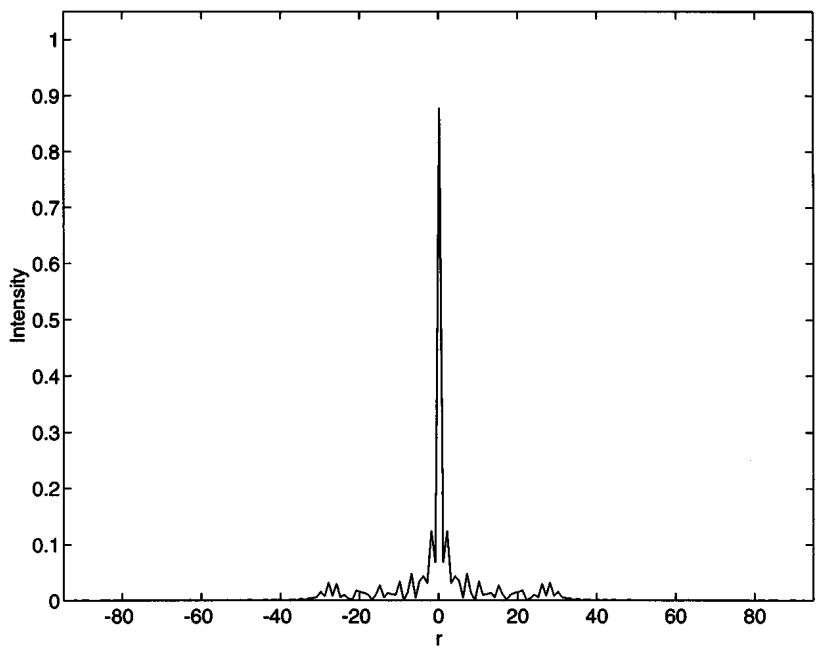

(c)

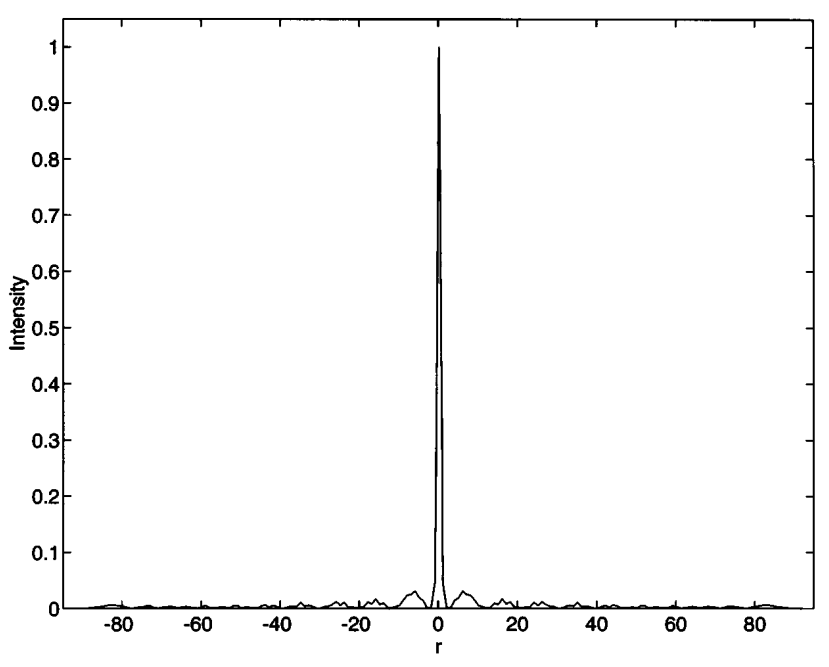

(e)

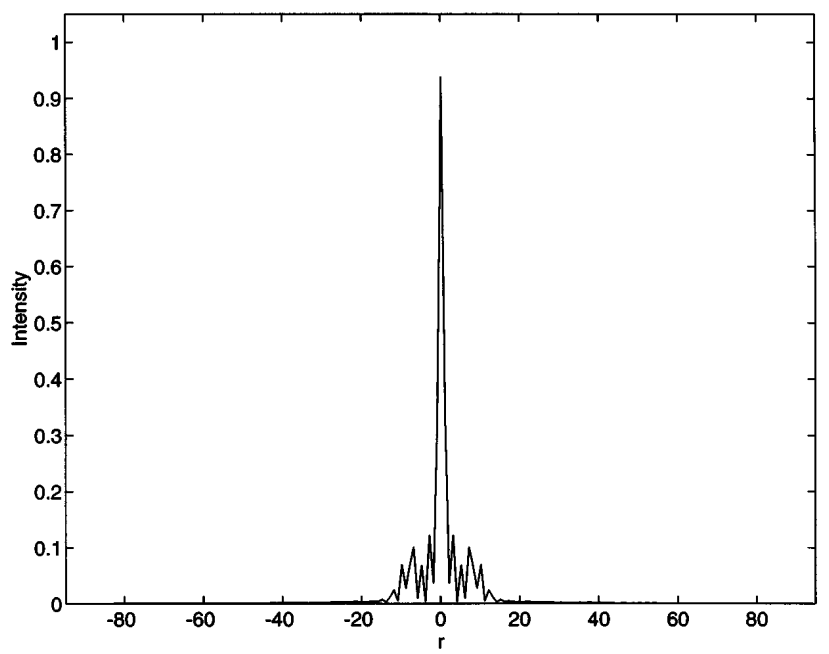

(b)

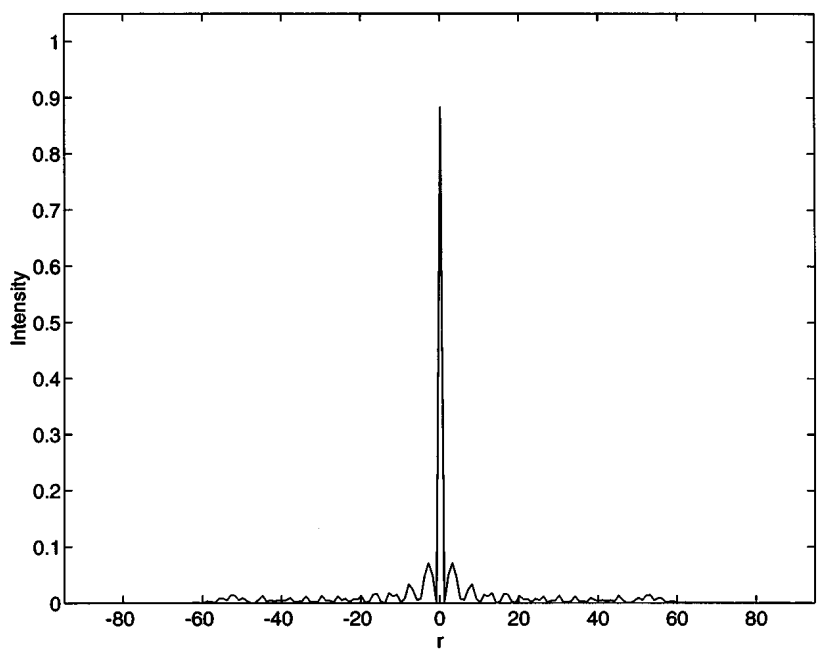

(d)

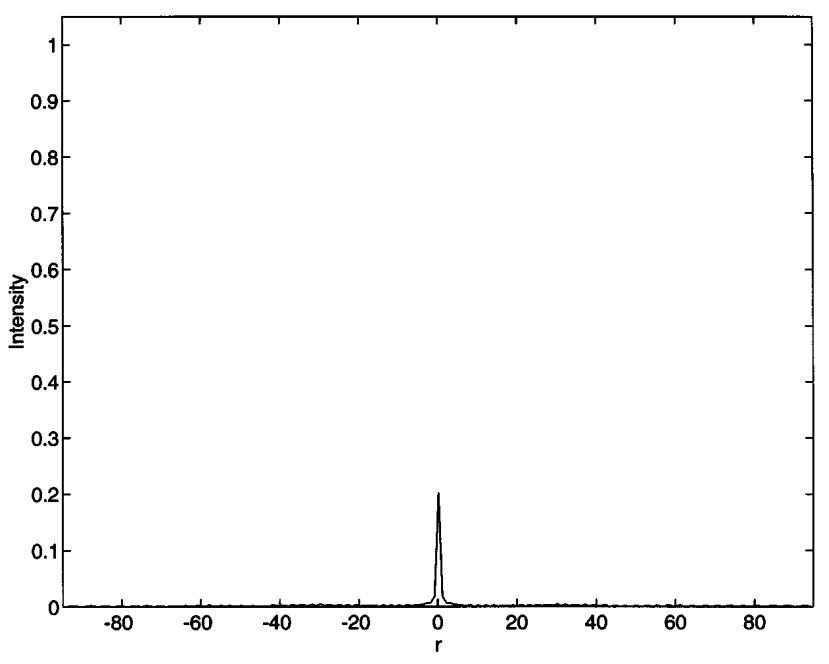

(f)

Fig. 3. Transverse cross section of the PNDB at (a) $z=0$, (b) $z=500$, (c) $z=1000$, (d) $z=1500$, (e) $z=2000$, and (f) $z=2500$. 


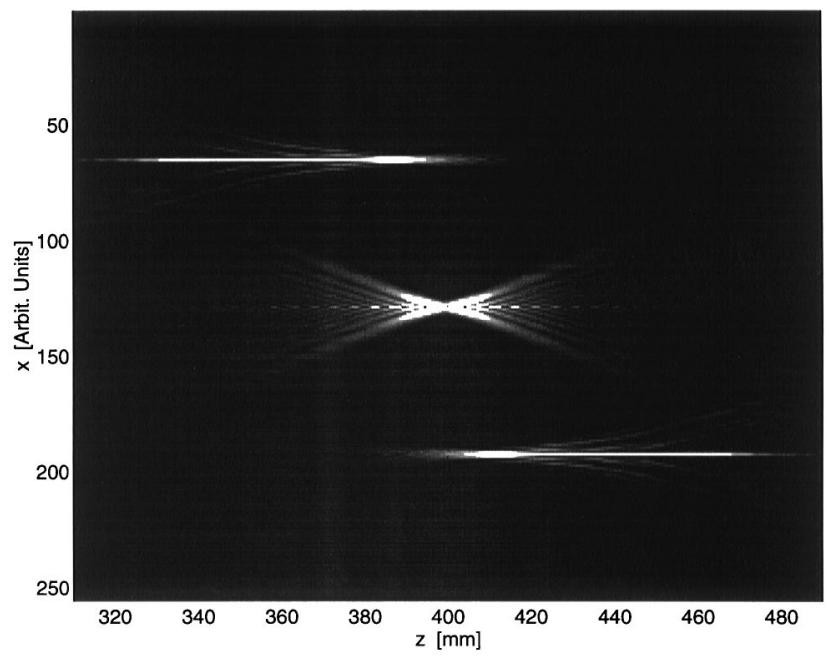

(a)

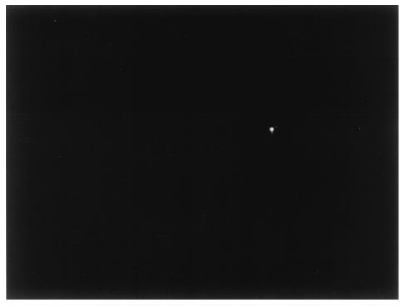

(b)

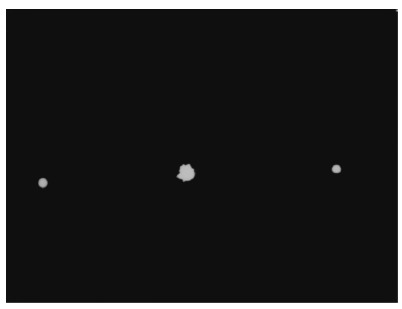

(d)

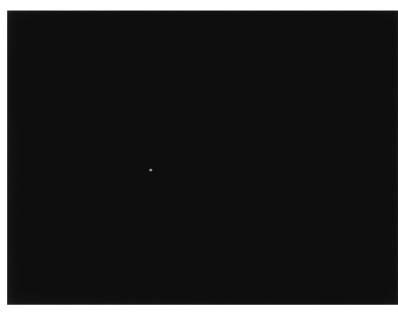

(f)

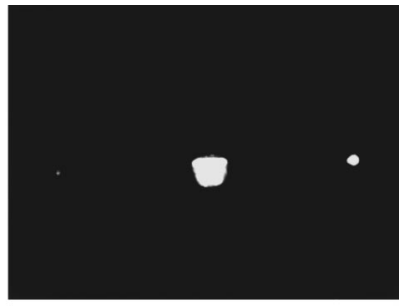

(c)

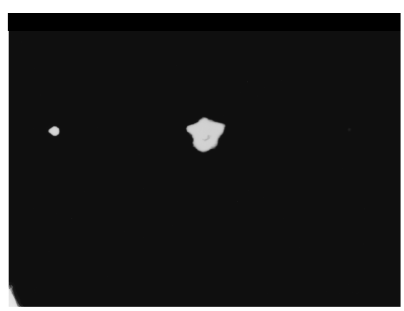

(e)

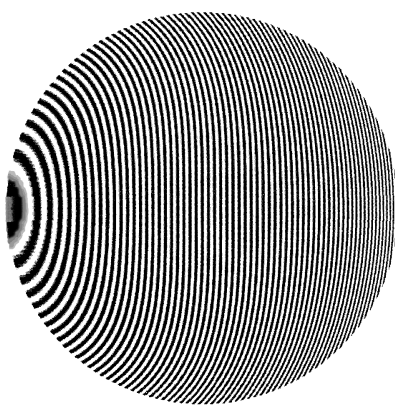

(g)
Fig. 4. (a) Output intensity distribution in the $x-z$ plane obtained by illumination of the hologram shown in (g) with a plane wave. Transverse cross sections are shown at distances (b) $33.5 \mathrm{~cm}\left(z=-z_{2}\right)$, (c) $38.5 \mathrm{~cm}\left(z=-z_{1}\right)$, (d) $40 \mathrm{~cm}(z=0)$, (e) $41.5 \mathrm{~cm}\left(z=z_{1}\right)$, and (f) $46.5 \mathrm{~cm}\left(z=z_{2}\right)$ from the lens (g) The Fourier hologram that generates this beam. The hologram's distribution is given in Eq. (25), where $d_{x}=5.13 \mathrm{~mm}^{-1}$, $b=3.72 \mathrm{~mm}$, and $R_{0}=6.2 \mathrm{~mm}$.

\section{EXPERIMENTAL RESULTS}

In the experimental part of this work we demonstrate the performance of the PNDB and the influence of chang- ing its parameters. Simultaneously, we illustrate the effects of some of the theorems presented in Section 3. The longitudinal beams that we use for this purpose are our proposed PNDB's, although these theorems are general and apply to any beam distribution. The optical system

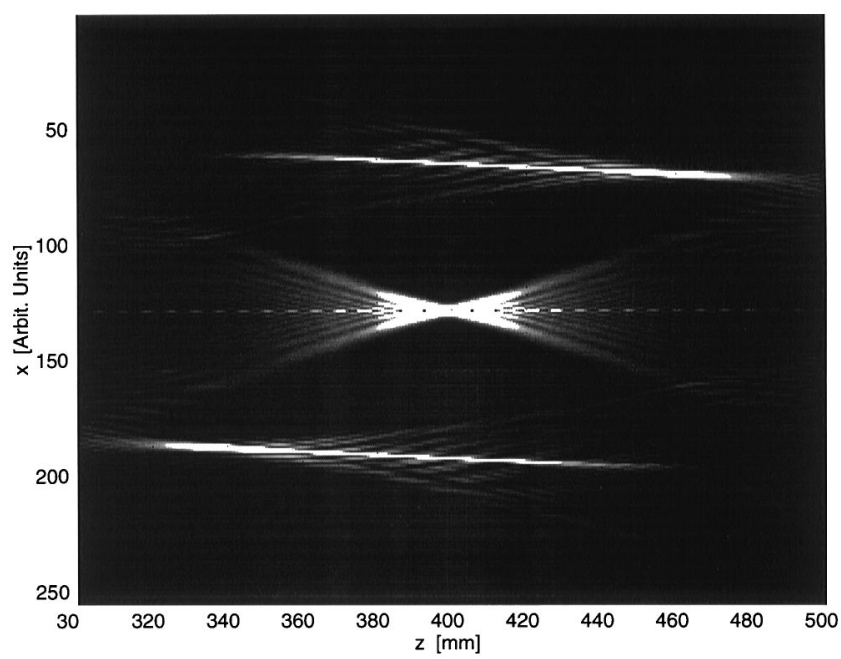

(a)

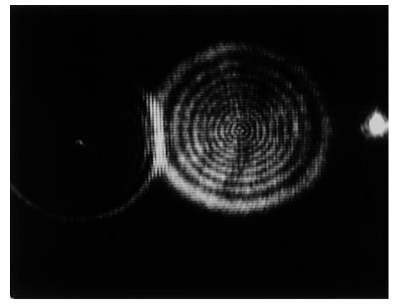

(b)

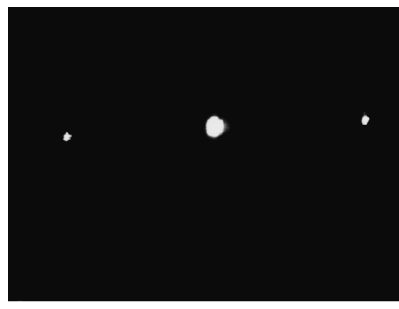

(d)

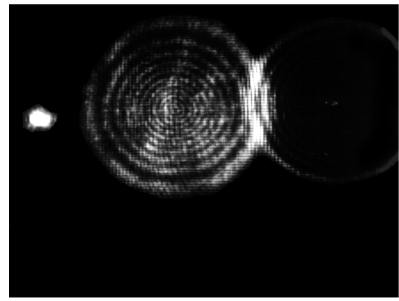

(f)

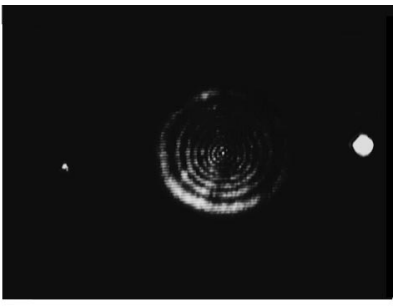

(c)

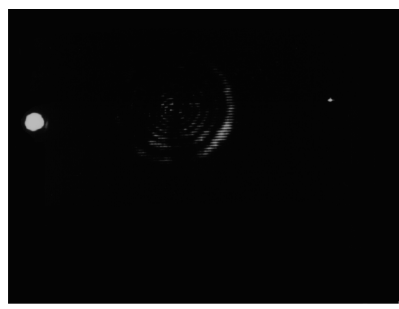

(e)

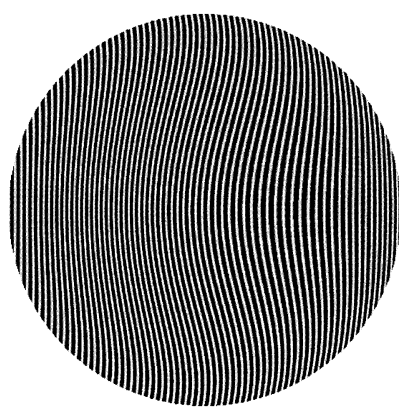

(g)
Fig. 5. As Fig. 4 with $\alpha=-0.31 \mathrm{~mm}^{-2}, d_{a}=20 \mathrm{~mm}, b=$ $3.6 \mathrm{~mm}, d_{x}=5.13 \mathrm{~mm}^{-1}$, and $R_{0}=6.2 \mathrm{~mm}$. Transverse cross sections are shown at distances (b) $34 \mathrm{~cm}$, (c) $35.5 \mathrm{~cm}$, (d) $40 \mathrm{~cm}$, (e) $44.5 \mathrm{~cm}$, and (f) $46 \mathrm{~cm}$ from the lens. (g) The Fourier hologram that generates this beam. 


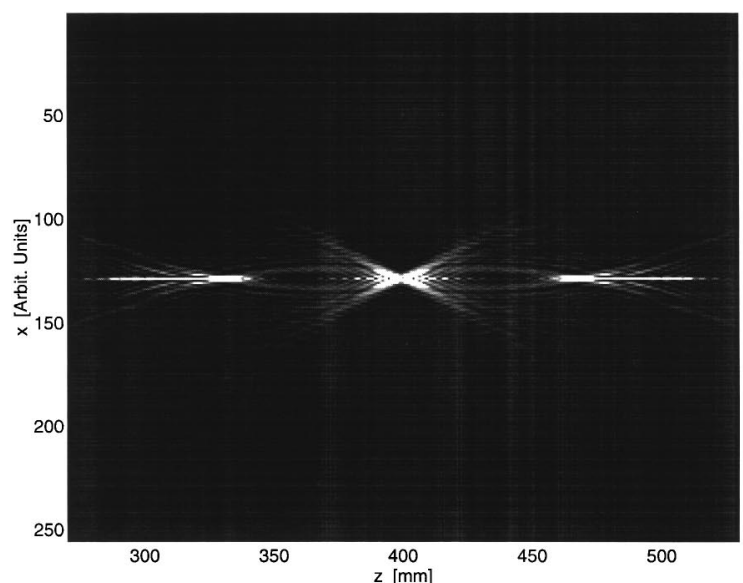

(a)

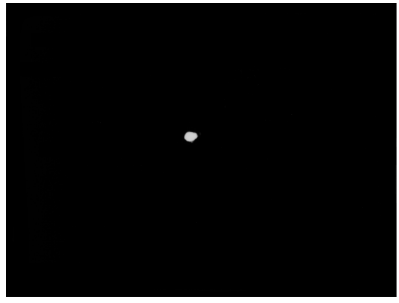

(d)

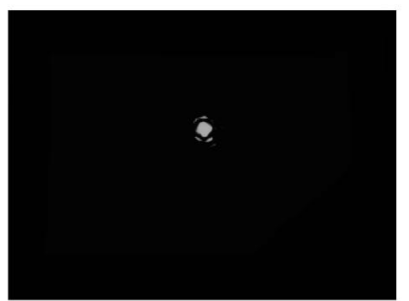

(h)

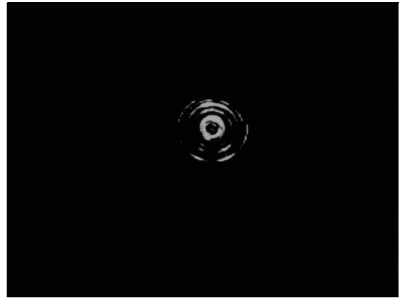

(e)

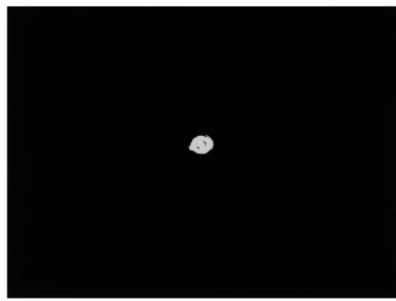

(i)

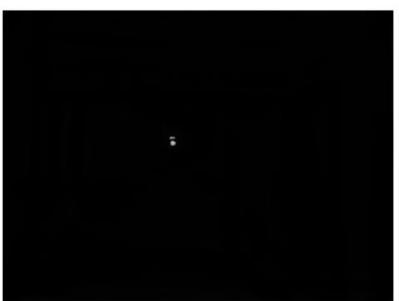

(b)

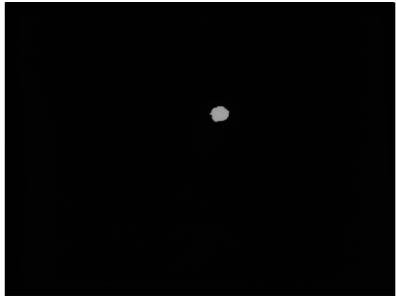

(f)

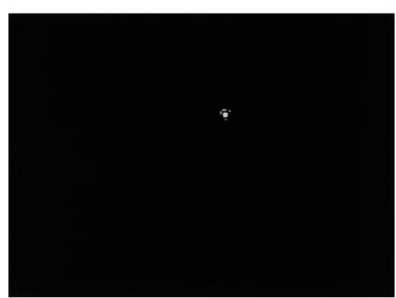

(j)

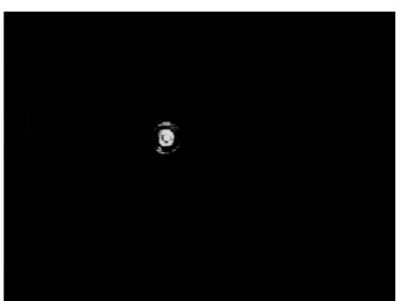

(c)

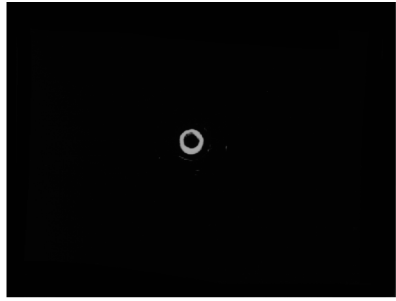

(g)

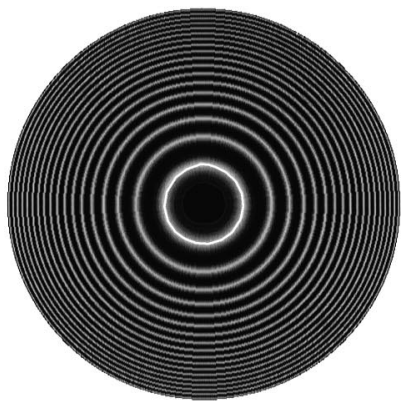

(k)

Fig. 6. As Fig. 4 with $\alpha=0.31 \mathrm{~mm}^{-2}, b=4 \mathrm{~mm}, d_{a}=d_{x}=0$, and $R_{0}=6.2 \mathrm{~mm}$. Transverse cross sections are shown at distances (b) $28 \mathrm{~cm}$, (c) $29.5 \mathrm{~cm}$, (d) $33 \mathrm{~cm}$, (e) $37.5 \mathrm{~cm}$, (f) $40 \mathrm{~cm}$, (g) $44.5 \mathrm{~cm}$, (h) $47 \mathrm{~cm}$, (i) $49.5 \mathrm{~cm}$, and (j) $52 \mathrm{~cm}$ from the lens. (k) The Fourier hologram that generates this beam.

that we use in this experiment is shown in Fig. 1, where $\lambda=0.6328 \mu \mathrm{m}$ and $f=40 \mathrm{~cm}$ for all the experiments.

The method we use to implement the RHF's is based on two of the above-mentioned theorems. Consequently, the RHF can be coded on a simple binary $\{0,1\}$, transparency, and the use of a complicated, expensive phase mask is avoided. However, we are aware of the fact that with use of the coding method, the obtained PNDB is just one term among three, and therefore the optical efficiency of the beam is much lower compared with that of the beam generated by a phase mask.

The hologram $h\left(x_{i}, y_{i}\right)$ in the first experiment is given by

$$
\begin{aligned}
h\left(x_{i}, y_{i}\right)= & \frac{1}{2}+\frac{1}{4} g_{4}\left(r_{i}\right) \exp \left(j 2 \pi d_{x} x_{i}\right) \\
& +\frac{1}{4} g_{4}^{*}\left(r_{i}\right) \exp \left(-j 2 \pi d_{x} x_{i}\right),
\end{aligned}
$$

where $d_{x}=5.13 \mathrm{~mm}^{-1}, b=3.72 \mathrm{~mm}$, and $R_{0}=6.2 \mathrm{~mm}$. Assuming that $\left|g_{4}\left(r_{i}\right)\right| \leq 1$, we realize that $h\left(x_{i}, y_{i}\right)$ is a positive real function. Using the halftone screening method in order to print out $h\left(x_{i}, y_{i}\right)$ from the computer, the gray-level function $h\left(x_{i}, y_{i}\right)$ is converted into a binary $\{0,1\}$ distribution. Note that $h$ contains the RHF and its conjugate, each of them multiplied by a linear phase

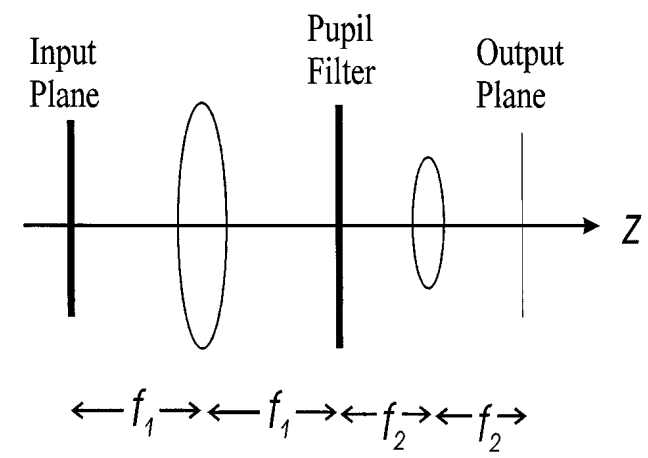

Fig. 7. Schematic imaging system with extended depth of focus. 
factor. On the basis of theorems 1 and 2, we expect to obtain two PNDB's, one existing in the interval $\left[z_{1}, z_{2}\right]$ and laterally shifted by the distance $\lambda f d_{x}$ from the optical axis, and the other existing in the interval $\left[-z_{2},-z_{1}\right]$ and laterally shifted by the distance $-\lambda f d_{x}$ from the optical axis. The solution is shown in Figs. 4(a)-4(f), and the generating hologram is depicted in Fig. $4(\mathrm{~g})$.

In the next experiment we demonstrate theorems 1-4 together, and for that purpose the computed hologram is

$$
\begin{aligned}
h\left(x_{i}, y_{i}\right)= & \frac{1}{2}+\frac{1}{4} g_{4}\left(x_{i}-d_{a}, y_{i}\right) \exp \left[j 2 \pi\left(d_{x} x_{i}+\alpha r_{i}^{2}\right)\right] \\
& +\frac{1}{4} g_{4}{ }^{*}\left(x_{i}-d_{a}, y_{i}\right) \exp \left[-j 2 \pi\left(d_{x} x_{i}+\alpha r_{i}{ }^{2}\right)\right],
\end{aligned}
$$

where $\alpha=-0.31 \mathrm{~mm}^{-2}, d_{a}=20 \mathrm{~mm}, b=3.6 \mathrm{~mm}$, and other parameters, $d_{x}$ and $R_{0}$, are as in the previous example. According to theorem 3 the two PNDB's should be shifted along their axes at a distance $2 \lambda f^{2} \alpha$ from the focal point. Following theorem 4, the beam should rotate an angle $\theta_{0}=\tan ^{-1}\left(d_{0} / f\right)=2.86^{\circ}$ in the $x-z$ plane around the points $\left(\lambda f d_{x}, 0\right)$ for one beam and $\left(-\lambda f d_{x}, 0\right)$ for the other. These effects are demonstrated clearly in Figs. 5(a)-5(f), and the corresponding hologram is shown in Fig. $5(\mathrm{~g})$.

Using theorem 3 , we can produce a real positive hologram distribution, even without introducing a linear phase factor, as in the following example:

$$
\begin{aligned}
h\left(x_{i}, y_{i}\right)= & \frac{1}{2}+\frac{1}{4} g_{4}\left(r_{i}\right) \exp \left[j 2 \pi \alpha{r_{i}}^{2}\right] \\
& +\frac{1}{4} g_{4}{ }^{*}\left(r_{i}\right) \exp \left[-j 2 \pi \alpha r_{i}{ }^{2}\right],
\end{aligned}
$$

where $b=4 \mathrm{~mm}, R_{0}=6.2 \mathrm{~mm}, \alpha=0.31 \mathrm{~mm}^{-2}$, and $d_{a}=$ $d_{x}=0$. This time, as we see in Figs. 6(a) $-6(\mathrm{j})$, the three

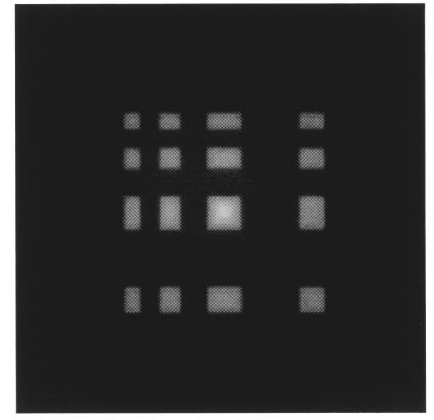

(a)

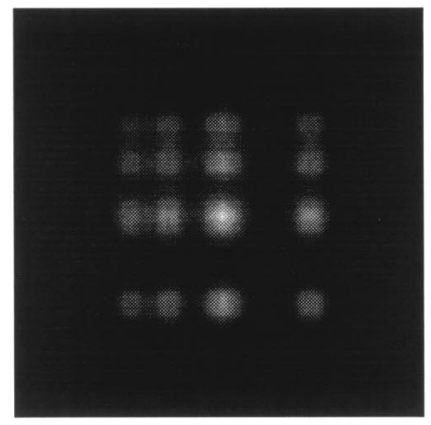

(d)

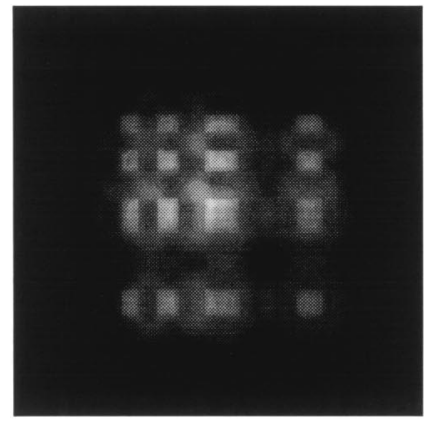

(g)

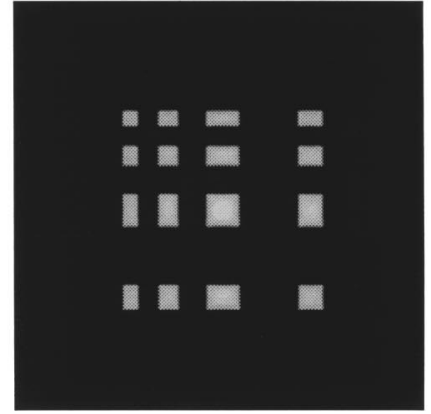

(b)

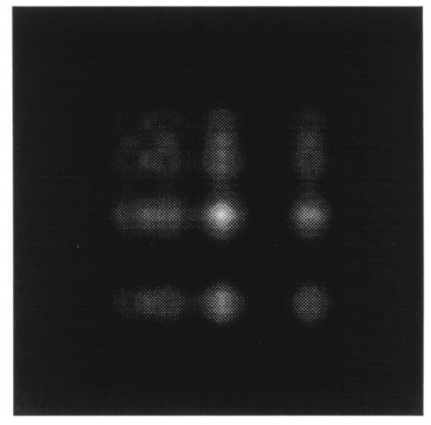

(e)

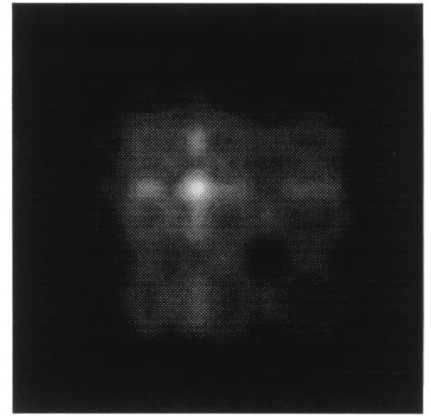

(h)

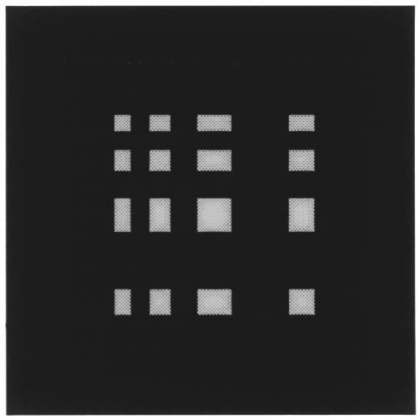

(c)

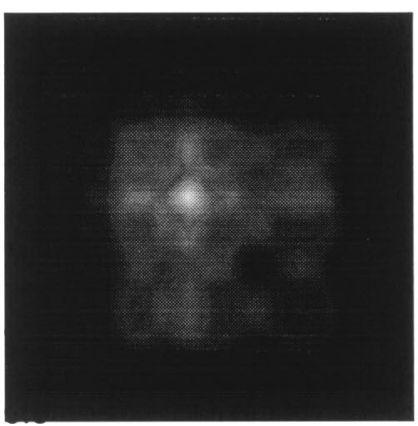

(f)

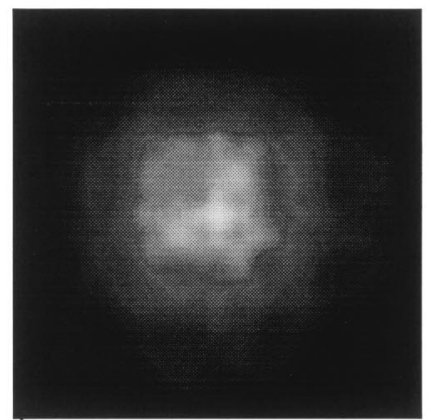

(i)

Fig. 8. Imaging results of three kinds of imaging system: with pupil filtering at (a) $z=0$, (d) $z=64$ pixels, and (g) $z=128$ pixels; with a clear pupil of diameter $2 b$ at (b) $z=0$, (e) $z=64$ pixels; and (h) $z=128$ pixels; and with a clear pupil of diameter $2 R_{0}$ at (c) $z=0$, (f) $z=64$ pixels, and (i) $z=128$ pixels. 
diffraction orders are distributed along the optical axis. The intensity distribution was generated by illumination of the hologram shown in Fig. 6(k).

Our last demonstration illustrates one possible application of the RHF as an element for increasing the depth of focus of an imaging system. Assume that we have a telescopic imaging system and that the RHF is displayed in its pupil (spatial-frequency) plane, as is shown in Fig. 7. As a result of an increase in the system's depth of focus, the image is obtained in focus and with the same size, along an axial interval equal to the extended depth of focus. If the RHF, with $p=4$, is chosen, the imaging system is lossless, in contrast to some other pupil-filtering methods. ${ }^{14}$ It should also be mentioned that the axicon ${ }^{1}$ and the axilens ${ }^{3}$ extend the depth of focus of an imaging system without losses. However, since each of them is basically a single-lens imaging system, the size of the in-focus image changes along the depth of the field. ${ }^{15}$

The simulation results of a spatially incoherent monochromatic imaging system are presented in Fig. 8. Three systems were checked: (1) a system with the RHF ( $p=4, b=64$ pixels, $R_{0}=128$ pixels); (2) a system without any filter and with a pupil diameter $2 b$ (this system has the same resolution as the first one); (3) a system without any filter and with pupil diameter $2 R_{0}$. This system is without losses, as is the first one. The output results from the systems are sampled in three planes along the optical axis. The first plane [Figs. 8(a)-(c)] is the image plane $(z=0)$, and all three images are seen clearly. Examining the output at a distance $z=64$ pixels [Figs. 8(d) $-(\mathrm{f})$ ], we see that systems 1 and 2 yield clear images, whereas the output of system 3 is blurred. Finally, Figs. 8(g)-(i) depict the output at the plane $z=128$ pixels, where the details of the image of system 1 can be resolved and the other two images are blurred. Note that the overall intensity of systems 1 and 3 is equal, whereas that of system 2 is only $\sim 6 \%$ of the intensity of them. Comparing systems 1 and 2, we see that although they have the same resolution, the depth of focus of system 1 is 12 times longer, albeit at the cost of poorer contrast. The reason for poor contrast in the output of system 1 is the presence of significant sidelobes in the point-spread function of this system [see Fig. 3(e)].

\section{CONCLUSION}

In this paper we introduced a new kind of PNDB. This beam is generated by a Fourier hologram a short distance $\left(z_{1}\right)$ behind the front focal plane of a spherical lens. The generating functions of this beam are the RHF's. The RHF with $p=4$ is especially interesting because it creates a PNDB without absorbing light, i.e., the RHF in this case becomes a phase-only filter, and as a result the efficiency of generating the PNDB is maximized.

Changing specific parameters in the hologram enables us to steer the beam in space. These features were expressed formally by a set of theorems and were demonstrated experimentally. Finally, we demonstrated an important application for the $\mathrm{RHF}$ as a pupil filter in imaging systems. Using the phase-only filter enables us to build an efficient imaging system with a long depth of focus.

\section{APPENDIX A}

In this appendix we present the proofs of the theorems of Section 3.

\section{Complex-conjugate theorem: proof}

$$
\begin{aligned}
\operatorname{FST}\left\{g^{*}\left(x_{i}, y_{i}\right)\right\}= & \frac{\exp [j k(z+2 f)]}{j \lambda f} \int_{-\infty}^{\infty} \int_{-\infty}^{\infty} g^{*}\left(x_{i}, y_{i}\right) \\
& \times \exp \left[\frac{j 2 \pi\left(x x_{i}+y y_{i}\right)}{\lambda f}\right. \\
& \left.-\frac{j \pi z\left(x_{i}{ }^{2}+y_{i}{ }^{2}\right)}{\lambda f^{2}}\right] \mathrm{d} x_{i} \mathrm{~d} y_{i} \\
= & \exp (j 4 k f-j \pi) \\
& \times\left\{\frac{\exp [j k(-z+2 f)]}{j \lambda f}\right. \\
& \times \int_{-\infty}^{\infty} \int_{-\infty}^{\infty} g\left(x_{i}, y_{i}\right) \exp \left[\frac{-j 2 \pi\left(x x_{i}+y y_{i}\right)}{\lambda f}\right. \\
& \left.\left.-\frac{-j \pi z\left(x_{i}{ }^{2}+y_{i}{ }^{2}\right)}{\lambda f^{2}}\right] \mathrm{~d} x_{i} \mathrm{~d} y_{i}\right\} \\
= & \exp (j 4 k f-j \pi) u^{*}(-x,-y,-z) . \quad(\mathrm{A} 1)
\end{aligned}
$$

\section{Linear phase theorem: proof}

$$
\begin{aligned}
& \operatorname{FST}\left\{g\left(r_{i}\right) \exp \left[j 2 \pi\left(d_{x} x_{i}+d_{y} y_{i}\right)\right]\right\} \\
& =\frac{\exp [j k(z+2 f)]}{j \lambda f} \int_{-\infty}^{\infty} \int_{-\infty}^{\infty} g\left(x_{i}, y_{i}\right) \exp \left\{j \frac { 2 \pi } { \lambda f } \left[\left(x-\lambda f d_{x}\right) x_{i}\right.\right. \\
& \left.\left.\quad+\left(y-\lambda g d_{y}\right) y_{i}\right]\right\} \exp \left[\frac{-j \pi z\left(x_{i}^{2}+y_{i}^{2}\right)}{\lambda f^{2}}\right] \mathrm{d} x_{i} \mathrm{~d} y_{i} \\
& =u\left(x-\lambda f d_{x}, y-\lambda f d_{y}, z\right) .
\end{aligned}
$$

3. Quadratic phase theorem: proof

$$
\begin{aligned}
\operatorname{FST}\left\{g\left(r_{i}\right)\right. & \left.\exp \left(j 2 \pi \alpha r_{i}{ }^{2}\right)\right\}=\frac{\exp [j k(z+2 f)]}{j \lambda f} \\
& \times \int_{-\infty}^{\infty} \int_{-\infty}^{\infty} g\left(x_{i}, y_{i}\right) \exp \left[j \frac{2 \pi}{\lambda f}\left(x x_{i}+y y_{i}\right)\right] \\
& \times \exp \left[-j 2 \pi r_{i}^{2}\left(\frac{z}{2 \lambda f^{2}}-\alpha\right)\right] r_{i} \mathrm{~d} r_{i} \\
= & \exp \left(j 4 \pi f^{2} \alpha\right) u\left(x, y, z-2 \lambda f^{2} \alpha\right) .
\end{aligned}
$$

4. Lateral-shift theorem: proof

Let us calculate the field distribution at a new coordinate system $(\bar{x}, \bar{y}, \bar{z})$ defined by the following transformation:

$$
\begin{aligned}
& x=\bar{x} \cos \theta+\bar{z} \sin \theta, \\
& y=\bar{y} \cos \varphi+\bar{z} \sin \varphi, \\
& z=(\bar{z} \cos \theta-\bar{x} \sin \theta) \cos \varphi-\bar{y} \sin \varphi,
\end{aligned}
$$

where $\tan \theta=d_{a} / f$ and $\tan \varphi=d_{b} / f$. The FST of the laterally shifted $g\left(x_{i}, y_{i}\right)$ is 


$$
\begin{aligned}
& \operatorname{FST}\left\{g\left(x_{i}-d_{a}, y_{i}-d_{b}\right)\right\}=\frac{\exp [j k(z+2 f)]}{j \lambda f} \\
& \quad \times \int_{-\infty}^{\infty} \int_{-\infty}^{\infty} g\left(x_{i}-d_{a}, y_{i}-d_{b}\right) \exp \left[\frac{j 2 \pi\left(x x_{i}+y y_{i}\right)}{\lambda f}\right] \\
& \quad \times \exp \left[\frac{-j \pi z\left(x_{i}{ }^{2}+y_{i}{ }^{2}\right)}{\lambda f^{2}}\right] \mathrm{d} x_{i} \mathrm{~d} y_{i} \\
& =\frac{1}{j \lambda f} \exp \left\{j k\left[z\left(1-\frac{d_{a}^{2}+d_{b}{ }^{2}}{2 f^{2}}\right)+2 f+\frac{x d_{a}+y d_{b}}{f}\right]\right\} \\
& \times \int_{-\infty}^{\infty} \int_{-\infty}^{\infty} g(\hat{x}, \hat{y}) \exp \left\{j \frac{2 \pi}{\lambda f}\left[\hat{x}\left(x-\frac{z d_{a}}{f}\right)+\hat{y}\left(y-\frac{z d_{b}}{f}\right)\right]\right\} \\
& \times \exp \left(\frac{-j \pi z\left(\hat{x}^{2}+\hat{y}^{2}\right)}{\lambda f^{2}}\right) \mathrm{d} \hat{x} \mathrm{~d} \hat{y} \\
& =\exp \left\{j k\left[z\left(-\frac{d_{a}{ }^{2}+d_{b}{ }^{2}}{2 f^{2}}\right)+\frac{x d_{a}+y d_{b}}{f}\right]\right\}\left(x-\frac{z d_{a}}{f}, y\right. \\
& \left.\quad-\frac{z d_{b}}{f}, z\right) \cdot(\mathrm{A} 4)
\end{aligned}
$$

Since $d_{a}=f \tan \theta, d_{b}=f \tan \varphi$, and, assuming $\cos \theta \approx$ $\cos \varphi \approx 1$ the result is FST along the new $\bar{z}$ axis with a different scale factor and multiplied by a different linear phase function. The $\bar{z}$ axis is the axis tilted by angle $\theta$ to the $y-z$ plane and by the angle $\varphi$ to the $x-z$ plane. Therefore, we can finally write

$$
\begin{aligned}
& \operatorname{FST}\left\{g\left(x_{i}-d_{a}, y_{i}-d_{b}\right)\right\} \\
& =\exp \left\{-\frac{j k z}{2}\left(\tan ^{2} \theta+\tan ^{2} \varphi\right)+j k[x \tan \theta+y \tan \varphi]\right\}
\end{aligned}
$$$$
\times u(\bar{x} \sec \theta, \bar{y} \sec \varphi, \bar{z} \cos \theta \cos \varphi) .
$$

\section{Similarity theorem: proof}

$$
\begin{aligned}
\operatorname{FST}\left\{g\left(s r_{i}\right)\right\}= & \frac{\exp [j k(z+2 f)]}{j \lambda f} \int_{0}^{2 \pi} \int_{0}^{\infty} g\left(s r_{i}\right) \\
& \times \exp \left[j \frac{2 \pi}{\lambda f}\left(x x_{i}+y y_{i}\right)\right] \\
& \times \exp \left(-j \frac{k z r_{i}^{2}}{2 f^{2}}\right) r_{i} \mathrm{~d} r_{i} d \theta_{i} \\
= & \frac{\exp [j k(z+2 f)]}{j \lambda f s^{2}} \int_{0}^{2 \pi} \int_{0}^{\infty} g\left(\psi_{i}\right) \\
& \times \exp \left[j \frac{2 \pi}{\lambda f s}\left(x \psi_{i} \cos \theta+y \psi_{i} \sin \theta\right)\right] \\
& \times \exp \left(-j \frac{k z \psi_{i}^{2}}{2 f^{2} s^{2}}\right) \psi_{i} \mathrm{~d} \psi_{i} d \theta_{i} \\
= & \frac{\exp \left[j k z\left(1-s^{-2}\right)\right]}{s^{2}} u\left(\frac{r}{s}, \frac{z}{s^{2}}\right),
\end{aligned}
$$

where $\psi_{i}=s r_{i}$.

6. Longitudinal-shift theorem: proof

When the hologram is displayed at a distance $d$ from the lens, the longitudinal distribution is calculated by a Fresnel transform from the input plane to the lens plane
$P_{L}$ and then by an additional Fresnel approximation from the lens plane to plane $P_{f}$, i.e.,

$$
\begin{aligned}
\operatorname{FST}\left\{g\left(r_{i}\right) * \delta\left(z_{i}-d\right)\right\}= & \frac{\exp \left\{j k\left[z+f+r^{2} / 2(z+f)\right]\right\}}{j \lambda(z+f)} \\
& \times \int_{0}^{2 \pi} \int_{0}^{\infty} g_{L}\left(x_{L}, y_{L}\right) \exp \{-j \\
& \times \frac{k z r_{L}^{2}}{2}\left[\frac{1}{f}-\frac{1}{(z+f)}\right] \\
& \left.+j \frac{k\left(x x_{L}+y y_{L}\right)}{z+f}\right\} r_{L} \mathrm{~d} r_{L} \mathrm{~d} \theta_{L},
\end{aligned}
$$

where $g_{L}\left(x_{L}, y_{L}\right)$ is the distribution right before the lens, given by

$$
\begin{aligned}
g_{L}\left(x_{L}, y_{L}\right)= & \frac{\exp (j k d)}{j \lambda d} \int_{-\infty}^{\infty} \int_{-\infty}^{\infty} g\left(x_{i}, y_{i}\right) \exp \left[-j \frac{k}{2 d}\left(r_{L}{ }^{2}+r_{i}{ }^{2}\right.\right. \\
& \left.\left.-2 x_{i} x_{L}-2 y_{i} y_{L}\right)\right] \mathrm{d} x_{i} \mathrm{~d} y_{i} .
\end{aligned}
$$

Substituting Eq. (A8) into Eq. (A7) and performing straightforward algebra yield

$$
\begin{aligned}
\operatorname{FST}\left\{g\left(r_{i}\right) *\right. & \left.\delta\left(z_{i}-d\right)\right\}=\frac{f \exp [j k(z+d+f)]}{j \lambda\left[z(f-d)+f^{2}\right]} \\
& \times \int_{-\infty}^{\infty} \int_{-\infty}^{\infty} g\left(x_{i}, y_{i}\right) \exp \{-j k \\
& \left.\times \frac{z\left[r_{i}^{2}+(f-d) r^{2}\right]-2 f\left(x x_{i}+y y_{i}\right)}{2\left[z(f-d)+f^{2}\right]}\right\} \mathrm{d} x_{i} \mathrm{~d} y_{i} \\
= & \frac{f^{2}}{z(f-d)+f^{2}} \exp (j k\{z+d-f \\
& \left.\left.+\frac{(f-d) r^{2}-2 f^{2} z}{2\left[z(f-d)+f^{2}\right]}\right\}\right) u(\tilde{x}, \tilde{y}, \tilde{z}),
\end{aligned}
$$

where

$$
(\tilde{x}, \tilde{y}, \tilde{z})=\frac{f^{2}}{z(f-d)+f^{2}}(x, y, z) .
$$

\section{ACKNOWLEDGMENTS}

This research was supported by the U.S. Army Research Office and the Advanced Research Projects Agency.

\section{REFERENCES}

1. J. H. McLeod, "The axicon: a new type of optical element," J. Opt. Soc. Am. 44, 592-597 (1954).

2. J. Durnin, "Exact solutions for diffraction-free beams. I. The scalar theory," J. Opt. Soc. Am. A 4, 651-654 (1987).

3. N. Davidson, A. A. Friesem, and E. Hasman, "Holographic axilens: high resolution and long focal depth," Opt. Lett. 16, 523-525 (1991); J. Sochacki, S. Bará, Z. Jaroszewicz, and A. Kołodziejczyk, "Phase retardation of the uniformintensity axilens," Opt. Lett. 17, 7-9 (1992).

4. J. Rosen, "Synthesis of nondiffracting beams in free space," Opt. Lett. 19, 369-371 (1994).

5. J. Rosen and A. Yariv, "Synthesis of an arbitrary axial field profile by computer-generated holograms," Opt. Lett. 19, 843-845 (1994). 
6. R. Piestun and J. Shamir, "Control of wave-front propagation with diffractive elements," Opt. Lett. 19,771-773 (1994).

7. J. Rosen, B. Salik, A. Yariv, and H.-K. Liu, "Pseudonondiffracting slitlike beam and its analogy to the pseudonondispersing pulse," Opt. Lett. 20, 423-425 (1995).

8. B. Salik, J. Rosen, and A. Yariv, "One-dimensional beam shaping,” J. Opt. Soc. Am. A 12, 1702-1706 (1995).

9. J. Durnin, J. J. Miceli, Jr., and J. H. Eberly, "Diffraction-free beams," Phys. Rev. Lett. 58, 1499-1501 (1987).

10. C. W. McCutchen, "Generalized aperture and the threedimensional diffraction image," J. Opt. Soc. Am. 54, 240-244 (1964).

11. J. Rosen and A. Yariv, "Snake beam: a paraxial arbitrary focal line," submitted to Opt. Lett.

12. A. Papoulis, Systems and Transforms with Applications in Optics (McGraw-Hill, New York, 1968), Chap. 7, pp. $222-254$.
13. R. M. Herman and T. A. Wiggins, "Apodization of diffractionless beams," Appl. Opt. 31, 5913-5915 (1992).

14. W. T. Welford, "Use of annular apertures to increase focal depth,” J. Opt. Soc. Am. 50, 749-753 (1960); H. Fukuda, T. Terasawa, and S. Okazaki, "Spatial filtering for depth of focus and resolution enhancement in optical lithography," J. Vac. Sci. Technol. B 9, 3113-3116 (1991); R. M. Von Bünau, G. Owen, and R. F. Pease, "Optimization of pupil filters for increased depth of focus,” Jpn. J. Appl. Phys. 32, 5850-5855 (1993).

15. J. Sochacki, A. Kołodziejczyk, Z. Jaroszewicz, and S. Bará, "Nonparaxial design of generalized axicons," Appl. Opt. 31, 5326-5330 (1992). 